\title{
Don't Forget the Cardiac Fibroma: Coronavirus Disease 2019 and the Management of Patient with a Large Cardiac Fibroma
}

\author{
Weston Andrews ${ }^{1}$, Thomas Moon ${ }^{2}$, Sunita Ferns ${ }^{3}$, Jeremy Gebhardt ${ }^{3}$, Kara Blackmer ${ }^{3}$, \\ Harma Turbendian ${ }^{4}$, and Michael Shillingford ${ }^{4}$ \\ ${ }^{1}$ University of Florida Health at Jacksonville \\ ${ }^{2}$ Division of Pediatric Cardiology, University of Florida College of Medicine \\ ${ }^{3}$ Wolfson Children's Hospital, Baptist Medical Center \\ ${ }^{4}$ Wolfson Children's Hospital, University of Pittsburgh Medical Center
}

July 26, 2021

\begin{abstract}
COVID-19 has introduced a monumental challenge to the routine delivery of healthcare and protection of healthcare professionals. Conditions that are life threatening and need urgent intervention add another layer of complexity. This brief report reviews the management of a large cardiac fibroma found in the setting of COVID-19.
\end{abstract}

Don't Forget the Cardiac Fibroma: Coronavirus Disease 2019 and the Management of Patient with a Large Cardiac Fibroma

Weston G. Andrews MD*, Thomas Moon MD**, Sunita Ferns MD***, Jeremy Gebhardt PA-C***, Kara Blackmer PA-C***, Harma K.Turbendian MD****, Michael Shillingford MD****

*Department of Surgery, University of Florida College of Medicine, Jacksonville, Florida

**Department of Pediatrics, Division of Pediatric Cardiology, University of Florida College of Medicine, Jacksonville, Florida

*** Wolfson Children's Hospital, Baptist Medical Center, Jacksonville, Florida

**** Wolfson Children's Hospital, University of Pittsburgh Medical Center, Jacksonville, Florida

Running Title: Covid-19 and a Pediatric Cardiac Fibroma

Corresponding Author:

Weston G. Andrews (ORCID: 0000-0003-3709-6838)

Department of Surgery UF College of Medicine - Jacksonville 653 West 8th Street, FC12 3rd Floor, Faculty Clinic Jacksonville, FL 32209

(904) 962-2236

Weston.andrews@jax.ufl.edu

Key Words: pediatric cardiac surgery, cardiac tumors, SARS CoV2

Conflicts of Interest: None

Source of Funding : None 


\begin{abstract}
COVID-19 has introduced a monumental challenge to the routine delivery of healthcare and protection of healthcare professionals. Conditions that are life threatening and need urgent intervention add another layer of complexity. This brief report reviews the management of a large cardiac fibroma found in the setting of COVID-19.
\end{abstract}

\title{
Abbreviations:
}

Coronavirus disease 2019 (COVID-19)

Severe acute respiratory syndrome coronavirus 2 (SARS-CoV2)

Electrocardiogram (ECG)

\section{Introduction}

The coronavirus disease-2019 (COVID-19) pandemic has had an unprecedented impact on patients and patient care management worldwide, including the field of congenital cardiology and cardiac surgery. ${ }^{1}$ Traditional management algorithms are challenged by the unpredictable nature of COVID-19. This case report highlights an important, treatable diagnosis complicated by a concomitant COVID-19 infection.

Primary cardiac tumors in children are extremely rare. ${ }^{2-4}$ Most tumors are reported as case reports or as single institution case series. These are usually benign, and the most common pathology in the pediatric population is the rhabdomyoma, followed by fibromas, myxomas, teratomas, and hemangiomas. Up to $12 \%$ will be found incidentally but the rate of growth, location, and size of the tumor determines the clinical presentation. ${ }^{5}$ Most children will present with a one or more of the following symptoms: murmur, arrhythmia, cyanosis, dyspnea, or cardiac failure. ${ }^{6}$ Surgical resection remains the standard of care for primary cardiac tumors in children. Echocardiography and magnetic resonance imaging are the gold standard for diagnosing cardiac tumors; computed tomography can be a useful adjunct.

Pediatric cardiac fibromas most often arise from the left ventricle. ${ }^{7}$ Noninvasive, fibromas of the heart can exert pressure on vital parts of the heart including the coronary arteries and valves. A multicenter European study of pediatric primary cardiac tumors reported a $10 \%$ rate of arrhythmias associated with cardiac fibromas and a $20 \%$ overall rate of arrhythmias associated with all cardiac tumors. ${ }^{2}$ These rhythm disturbances were most often ectopic atrial or ventricular beats, followed by atrial flutter and paroxysmal supraventricular tachycardia. Due to the association with sudden cardiac death, pediatric cardiac fibromas are typically resected even in asymptomatic patients. ${ }^{8}$ We report a pediatric case of cardiac fibroma in a child who was diagnosed with severe acute respiratory syndrome coronavirus 2 (SARS-CoV2) on a pre-operative work-up. No institutional review, consent, or clinical trial registration was required for this case report and review.

\section{Case Report}

A 4-year-old male with no significant past medical history presented to his pediatrician for a routine wellchild visit and was noted to be in tachycardia at over 250 beats per minute. Further questioning revealed he had been experiencing some nausea and vomiting a few days prior to presentation but was otherwise asymptomatic. He was sent to the emergency room where an electrocardiogram (ECG) demonstrated a wide complex tachycardia at a rate of $270 \mathrm{bpm}$ (Figure 1). He received three escalating doses of adenosine with no effect, followed by amiodarone $(5 \mathrm{mg} / \mathrm{kg})$ intravenously with some rate but no rhythm control. He was then sedated and successfully cardioverted to a normal sinus rhythm. A chest radiograph showed a cardiac silhouette that appeared enlarged (Figure 2) and he was admitted for further management. Lab work was remarkable for a hemoglobin of $10.9 \mathrm{~g} / \mathrm{dL}$, brain natriuretic peptide $(\mathrm{BNP})$ of $998 \mathrm{pg} / \mathrm{mL}, \mathrm{C}$ reactive protein of $3.8 \mathrm{mg} / \mathrm{dL}$, and a troponin of $0.21 \mathrm{ng} / \mathrm{mL}$. The viral panel was positive for Epstein Barr Virus and Human Rhinovirus at presentation and was initially negative for the SARS CoV2 Rapid PCR test. A repeat PCR test 48 hours later, however, was positive for SARS CoV2. Echocardiogram images demonstrated a left ventricular (LV) mass along the free wall of the left ventricle extending to the apex and a small pericardial as 
well as a right pleural effusion. (Figure 3) The mass was further delineated on a cardiac magnetic resonance imaging scan that showed a 22x $52 \mathrm{~mm}$ mass with a distinct separation between the LV. The mass presumed to be a fibroma based on MRI characterization. (Figure 4)

He was started on $3 \mathrm{mg} / \mathrm{kg}$ /day of propranolol administered TID. He did not experience any further episodes of ventricular tachycardia and only had occasional premature ventricular contractions. Serial BNP and troponin labs began to normalize within 72 hours of admission. Considering the positive SARS CoV2 PCR test, cardiac surgery for mass excision was postponed. He was discharged home on a 30-day real-time, wireless cardiac event monitor that allowed for close monitoring while awaiting surgery.

Three weeks later he underwent a resection of the cardiac fibroma. The mass was intimately associated with the distal left anterior descending artery which was preserved and had limited septum involvement. He was transferred to the pediatric cardiovascular intensive care unit and had an uneventful recovery. A postoperative echocardiogram demonstrated normal biventricular function with no valve regurgitation and no residual mass or effusion. He was discharged home on post-operative day 5 after an uneventful postoperative course. The propranolol was eventually discontinued 3 months post-surgery as he continued to demonstrate no arrhythmias. The final pathology demonstrated a benign cardiac fibroma with intact borders.

\section{Discussion}

Pediatric cardiac fibromas require prompt surgical attention. Due to the less invasive and benign nature, surgical treatment is considered relatively safe, with low intraoperative and post-operative morbidity and mortality rates. ${ }^{2,7}$ The diagnosis itself is an indication for resection, but may also include the presence of cardiac, pulmonary, or neurogenic symptoms, abnormal ECG findings, and evidence of hemodynamic impairment on echocardiogram. The presentation of the reported cardiac fibroma is not uncommon. A 42-year experience with pediatric cardiac tumors from Boston Children's Hospital reported that, compared to other tumors of the heart, fibromas were more likely to present with clinically significant arrhythmias. ${ }^{9}$ This patient was successfully stabilized with cardioversion and managed medically while he was evaluated for surgical resection. Echocardiography and MRI allowed for the tumor margins and architecture to be assessed adequately and for a surgical strategy to be planned.

The concurrent diagnosis of COVID-19 added a layer of complexity. Fortunately, the only side-effect experienced was a delay in definitive treatment. While this novel virus is known to cause cardiac arrhythmias in adults, it is difficult to speculate as to whether the virus precipitated the tachyarrhythmia or whether the tachyarrhythmia associated with the cardiac fibroma might have occurred independent of the viral illness. ${ }^{10}$ Once the child was diagnosed with COVID-19, an alternative plan needed to be fashioned to ensure patient safety. There are no consensus guidelines for triaging this unique patient. ${ }^{11}$ The care team prioritized the immediate risk to his life; the arrhythmia secondary to the cardiac fibroma. Once stable on medical management, the child then was sent home with close follow-up to recover from a relative asymptomatic COVID-19 infection. The patient returned three weeks later with a negative test for COVID-19 and underwent surgical resection.

This case report highlights critical dilemmas faced by the health care community during this pandemic. How do we care for a patient with a life-threatening condition that concurrently presents with COVID-19? And how do we quantify the risk of exposing surgical and medical personnel to the risk of delaying treatment of a potentially lethal condition? A case such as this requires a large amount of specialized healthcare personnel to care for the patient. The risk to healthcare workers is significant during this pandemic and has the potential to cripple a congenital cardiac program if one were to contract the virus. On the other hand, this child experienced a potentially malignant arrhythmia with a very treatable cause. Treatment decisions, especially in cases such as this, require a thoughtful risk benefit analysis and consensus by the entire treatment team. In summery we present the successful treatment of a pediatric cardiac fibroma in the setting of a COVID-19 diagnosis.

Figure 1: Electrocardiogram at presentation shows a wide complex tachycardia with atrioventricular dissociation at $270 \mathrm{bpm}$ with a right bundle branch block pattern, negative complexes in the inferior leads 
(II, III and aVF) and left sided leads (I, VL, V4-6) suggesting a more apical origin near the free wall of the left ventricle.

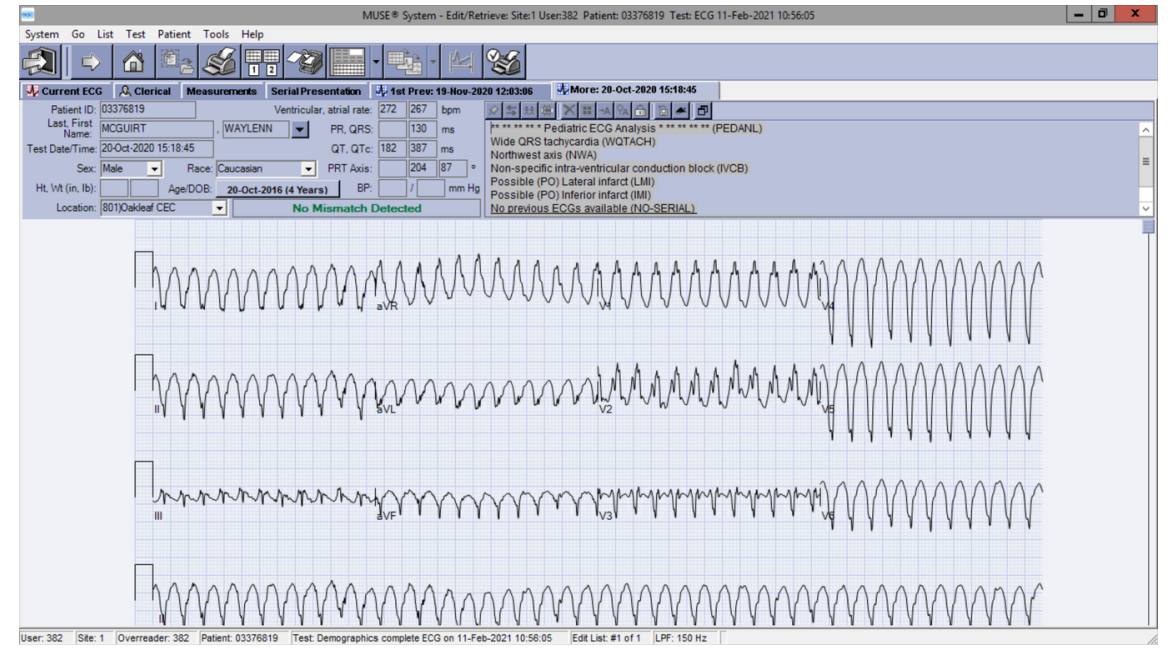

Figure 2 : Chest radiograph depicting left ventricular free wall fibroma

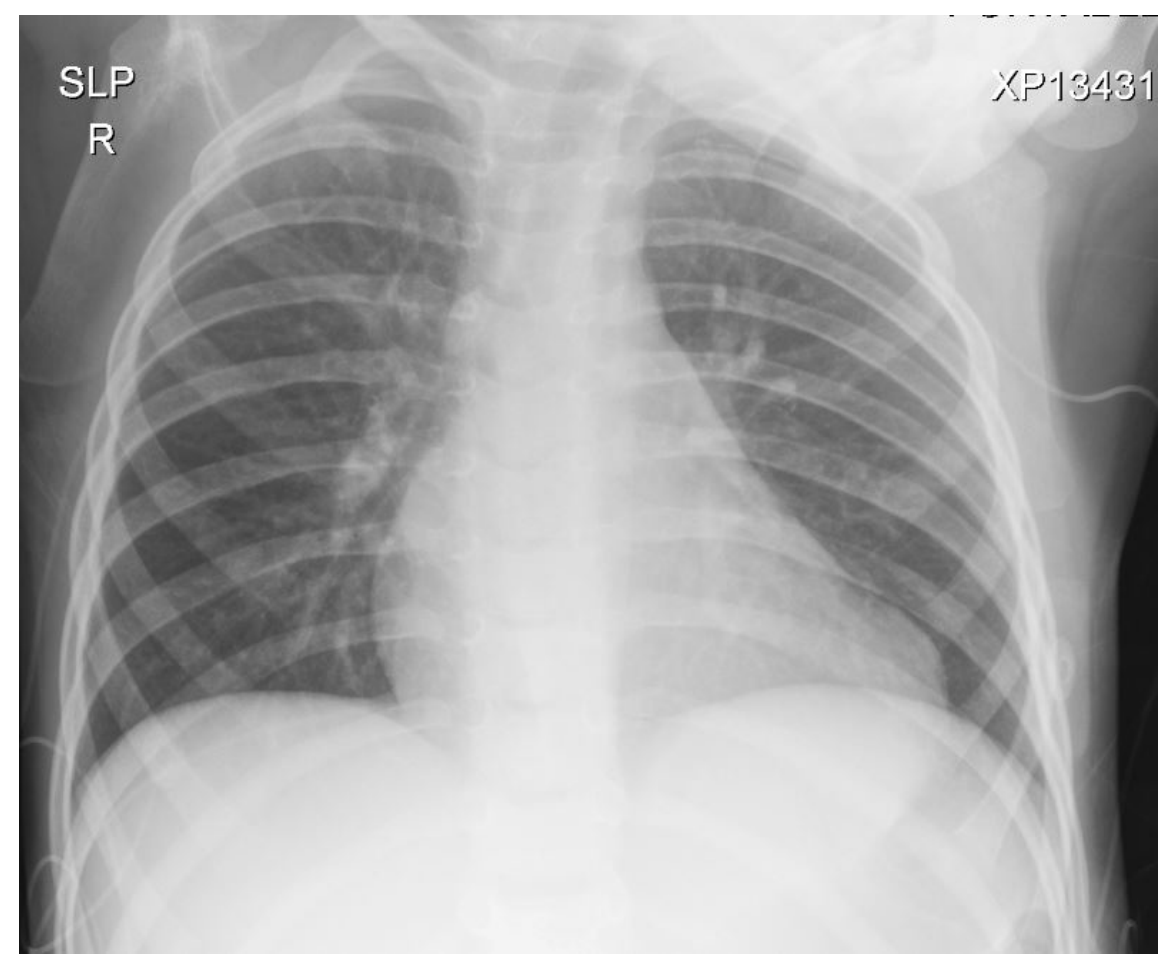

Figure 3 : Echocardiography of left ventricular free wall fibroma 


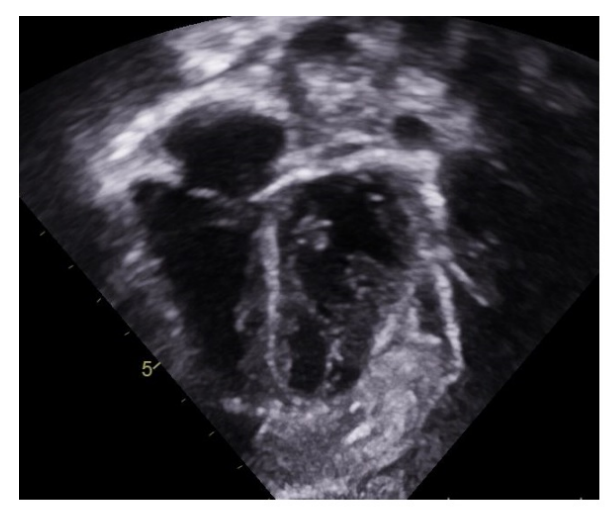

Figure 4 : Cardiac magnetic resonance imaging depicting a left ventricular free wall fibroma

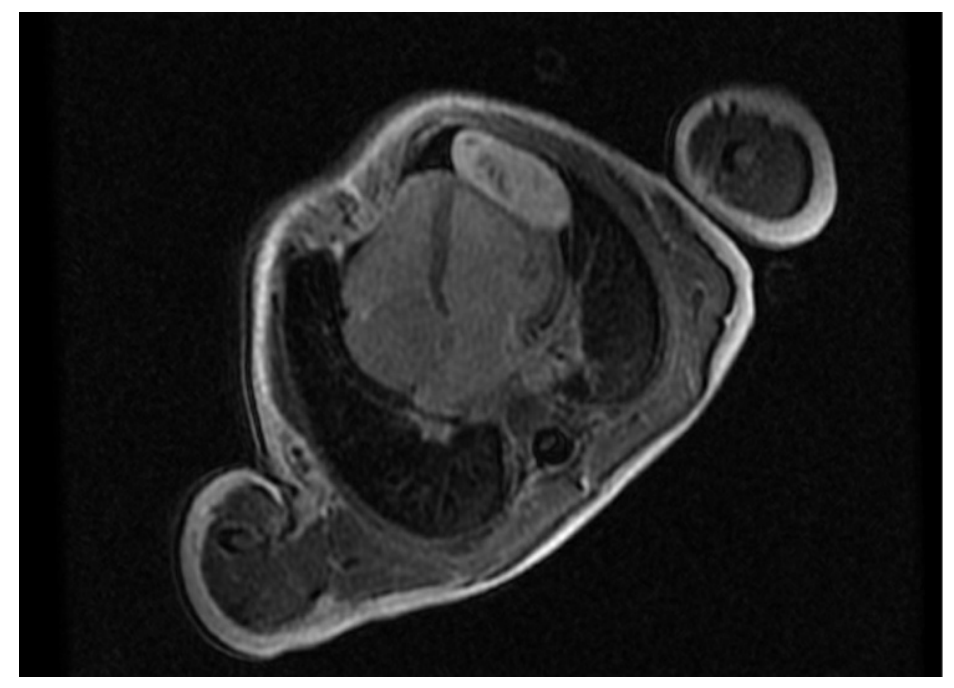

\section{References}

1. Protopapas EM, Rito M Lo, Vida VL, et al. Early Impact of the COVID-19 Pandemic on Congenital Heart Surgery Programs Across the World: Assessment by a Global Multi-Societal Consortium. World $J$ Pediatr Congenit Hear Surg . 2020;11(6):689-696. doi:10.1177/2150135120949462

2. Padalino MA, Vida VL, Boccuzzo G, et al. Surgery for primary cardiac tumors in children early and late results in a multicenter european congenital heart surgeons association study. Circulation . 2012;126(1):2230. doi:10.1161/CIRCULATIONAHA.111.037226

3. Delmo Walter EM, Javier MF, Sander F, Hartmann B, Ekkernkamp A, Hetzer R. Primary Cardiac Tumors in Infants and Children: Surgical Strategy and Long-Term Outcome. Ann Thorac Surg . 2016;102(6):20622069. doi:10.1016/j.athoracsur.2016.04.057

4. Ying L, Lin R, Gao Z, Qi J, Zhang Z, Gu W. Primary cardiac tumors in children: A center's experience. J Cardiothorac Surg . 2016;11(1):1-4. doi:10.1186/s13019-016-0448-5 
5. Reynen K. Cardiac Myxomas. N Engl J Med . 1995;333(24):1610-1617. doi:10.1056/NEJM199512143332407

6. Isaacs H. Fetal and neonatal cardiac tumors. Pediatr Cardiol . 2004;25(3):252-273. doi:10.1007/s00246003-0590-4

7. Cho JM, Danielson GK, Puga FJ, et al. Surgical Resection of Ventricular Cardiac Fibromas: Early and Late Results. Ann Thorac Surg . 2003;76(6):1929-1934. doi:10.1016/S0003-4975(03)01196-2

8. Cina SJ, Smialek JE, Burke AP, Virmani R, Hutchins GM. Primary cardiac tumors causing sudden death: A review of the literature.Am J Forensic Med Pathol . 1996;17(4):271-281. doi:10.1097/00000433199612000-00001

9. Miyake CY, Del Nido PJ, Alexander ME, et al. Cardiac tumors and associated arrhythmias in pediatric patients, with observations on surgical therapy for ventricular tachycardia. J Am Coll Cardiol . 2011;58(18):1903-1909. doi:10.1016/j.jacc.2011.08.005

10. Kochi AN, Tagliari AP, Forleo GB, Fassini GM, Tondo C. Cardiac and arrhythmic complications in patients with COVID-19. J Cardiovasc Electrophysiol . 2020;31(5):1003-1008. doi:10.1111/jce.14479

11. Stephens EH, Dearani JA, Guleserian KJ, et al. COVID-19: Crisis Management in Congenital Heart Surgery. Ann Thorac Surg . 2020;110(2):701-706. doi:10.1016/j.athoracsur.2020.04.001 